\title{
Surgical Preparation of a Permanent Carotid Transposition in Sheep
}

\author{
Liana V. Gouvêa, Ernane P. F. Novais, Ceci R. Leite, Fábio H. B. Ximenes, \\ Ricardo M. Almeida, R. B. Molás, J. G. C. Palermo, C. E. V. Silva, \\ Jose R. J. Borges, Eduardo M. M. Lima, Roberta Ferro Godoy \\ Large Animal Hospital, School of Veterinary Medicine, University of Brasilia, Brasilia, Brazil \\ E-mail: robertagodoy@unb.br
}

Received August 3, 2011; revised September 1, 2011; accepted September 10, 2011

\begin{abstract}
In large animal research, when frequent sampling of arterial blood is needed, the carotid artery transposition is the most used technique. The objective of this paper is to describe a new technique for carotid artery transposition in sheep and evaluate its effectiveness using the echo-Doppler ultrasound. The animals enrolled in this study had their carotid surgically elevated to the subcutaneous level, by suturing underneath muscles sternocephalic and brachiocephalic. None of the animals that underwent the procedure in this study had trans- or postoperative complications. Six months after the suture removal, all animals presented patent carotids by echo-Doppler ultrasound. This is an easy and safe procedure for carrying out experiments that require the collection of arterial blood.
\end{abstract}

Keywords: Blood Gas Analysis, Eco-Doppler, Experimental Surgery

\section{Introduction}

Easy and rapid access to large-caliber arteries are frequently needed in invasive studies about hemodynamics and/or blood gas analysis in large animals, especially if multiple samples are required. These arteries should be accessible, but protected from trauma, and their patency should be maintained for long periods [1]. Several descriptions of displacement of the carotid artery to the subcutaneous tissue of ruminants have been reported in the literature [2-8]. Although this is a well studied technique, a new choice for this procedure allowing a patent carotid artery for a prolonged time is presented, as well as minimum complication is observed.

In the technique described in [4], an incision is made parallel and ventral to the jugular vein, in the transition between the middle and distal third of the jugular groove. The carotid artery is located and a $10 \mathrm{~cm}$ section is externalized. The dorsal and the ventral margin of the esternocephalic and brachiocephalic muscles are sutured underneath the artery and above the jugular. The skin is sutured over the carotid artery. The disadvantage of this technique is that the carotid is not immobilized in the subcutaneous tissue, hindering the collection of arterial blood [1].
There is another described a technique [2] that differs from the previous by suturing the skin under de carotid artery, creating a skin tube containing the carotid artery. This technique requires wrapping the animal's neck to prevent accidental puncture of the artery. Problems were reported like skin necrosis and flow disturbances with this technique, and the first two studies have reported accidental carotid puncture [1,2,7].

A third technique described in [1], a modification of the technique originally proposed in [3]. The incision was made parallel and ventral to the jugular groove to allow the identification of the jugular vein. The skin is dissected, creating a flap which is folded dorsally. The carotid artery is then isolated and displaced as in the other techniques. The skin flap is returned to its original position and the skin on top of the exposed carotid artery is sutured around it. This technique has a lower risk of accidental perforation of the carotid due to the way that the artery is positioned.

A work [9] reported 94\% success rate after surgery relocation of carotid artery using the techniques from [2] and [3]. However, it reported also complications including skin necrosis and loss of arterial patency. The ideal technique would be able to maintain the viability of the carotid artery for a long period of time. The aim of this 
paper is to describe a new technique for transposition of the carotid artery in sheep and evaluate its effectiveness using the apparatus of ultrasound with echo-Doppler.

\section{Material and Methods}

This study was approved by the Ethics Committee on Animal Use (CEUA), at the University of Brasília (Brazil), under protocol 76850/2007.

\subsection{Experimental Group}

We used seven adult Santa Inês sheep with body mass between 36 and $42 \mathrm{~kg}$. Healthy status was determined by physical exam, complete blood count, biochemistry panel, and fecal flotation exam. Animals were kept in confinement and were fed twice a day with Tifton grass, water and salt ad libitum during the experiment. They were monitored daily pre and postoperatively by physical examination.

\subsection{Anaesthetic Procedure and Monitoring}

The animals were food fasted for 18 hours and water fasted 8 hours before the procedure. The premedication consisted of acepromazine (1\% Acepran, Univet S/A, São Paulo, Brazil) at the dose of $0.1 \mathrm{mg} / \mathrm{Kg}, \mathrm{IV}$. Anesthe- sia was induced with propofol (Propovan, Crystal, Itapira, Brazil) at the dose of $4.4 \mathrm{mg} / \mathrm{Kg}$ IV. The intubation time provided by this protocol was 5 minutes. The animal was intubated with the aid of a laryngoscope, endotracheal tube 7.5 with an extension and a guide wire.

Anesthesia was maintained with isoflurane (Isoforine, Crystal, Itapira, Brazil) in 100\% oxygen through a universal vaporizer and all animals were subjected to intermittent positive pressure ventilation (Conquest Slim Fan, HB Hospitalar, São Paulo, Brazil) with flow of 15 $\mathrm{mL} \cdot \mathrm{kg} \cdot \mathrm{min}^{-1} \mathrm{O}_{2}$. The airway pressure was kept within 20 $\mathrm{cm} \mathrm{H}_{2} \mathrm{O}$, with a tidal volume of $15 \mathrm{~mL} / \mathrm{kg}$ and $\mathrm{I}$ : $\mathrm{E}$ ratio was $1: 3$.

Monitors with heart rate (CardioPET, R \& D Mediq Ltda., São Paulo, Brazil) and $\mathrm{SpO}_{2}$ measurements (Active “ES”, Transform Advanced Technology Ltda., São Paulo, Brazil) were used to monitor the anesthesia. Respiratory rate and body temperature were also registered. Medium arterial pressure (MAP) was measured with vascular Doppler (Vet-Dop, VMED, Mill Creek, USA) positioned over the right metatarsal artery.

\subsection{Surgical Procedure}

The animals were positioned in left lateral recumbency. The animal's head was positioned partly outside the sur- gical bed providing a proper slope for drainage of the saliva produced, preventing its accumulation.

Due to the proximity of left common carotid artery to the esophagus, the right carotid artery was chosen for this procedure which is easier to be exposed. An area of about $15 \times 20 \mathrm{~cm}$ in the region of the jugular groove, in the right middle third of the neck, was shaved and prepared for surgery with Polyvinylpyrrolidone topic (Riodeine, Rioquímica Pharmaceutical Industry, São Jose do Rio Preto, Brazil) and iodine alcohol.

An incision of approximately $15 \mathrm{~cm}$ in a half-moon shape was made in the skin (Figure 1) involving the jugular groove. The skin and subcutaneous tissue was retracted for the identification of the jugular vein (Figure 2). The jugular was displaced dorsally to allow the observation of the carotid artery. In a segment of approximately $10 \mathrm{~cm}$ of the carotid artery, small branches from the artery were sutured with Vicryl 2 - 0 (Vicryl Ethicon, Johnson \& Johnson, São José dos Campos, Brazil) to prevent bleeding.

The vagosimpathetic branch passing close to the carotid artery was identified and carefully dissected dorsally to avoid injury. The dorsal margins of the esternocephalic muscle fascia and brachiocephalic ventral margin were sutured underneath the carotid and over the jugular vein with 2 - 0 Vicryl in a simple continuous pattern, keeping the segment of the artery near the skin and covering the jugular vein (Figure 3). Finally, the reduction of subcutaneous space was performed with Vicryl 2 - 0 and the epidermal suture with 0 nylon in a simple continuous pattern concluded the procedure.

\subsection{Post-Operative Care}

After surgery, the animals received cryotherapy for 15 minutes at the site of transposition, $10 \mathrm{mg} / \mathrm{animal}$ of

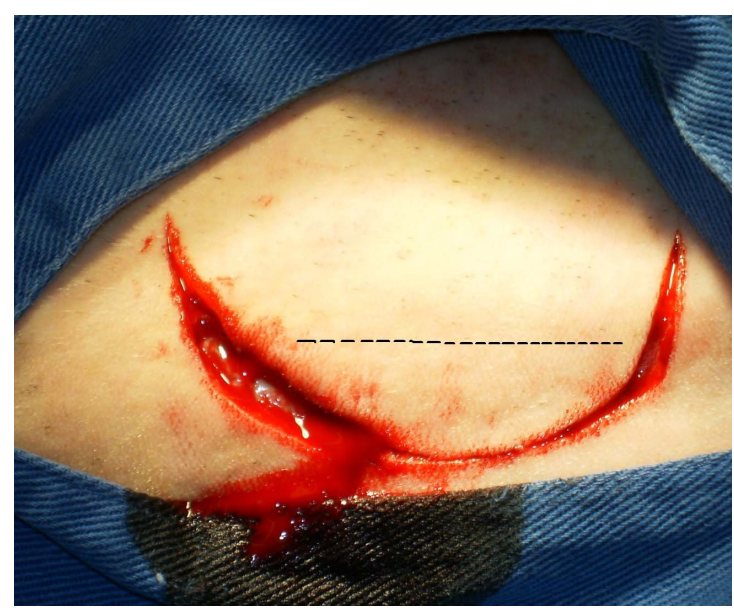

Figure 1. Incision in a half-moon shape in the skin surrounding the jugular groove (dashed line). 


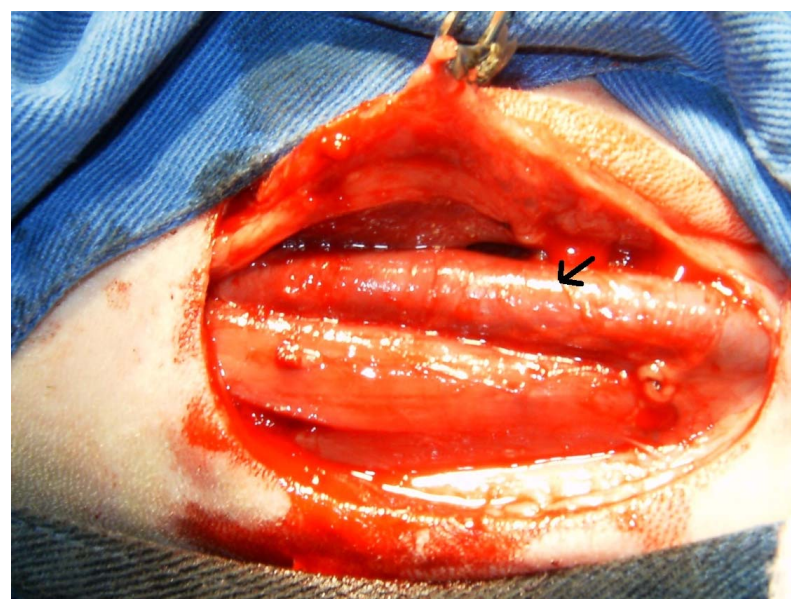

Figure 2. Identification of the jugular vein (black arrow).

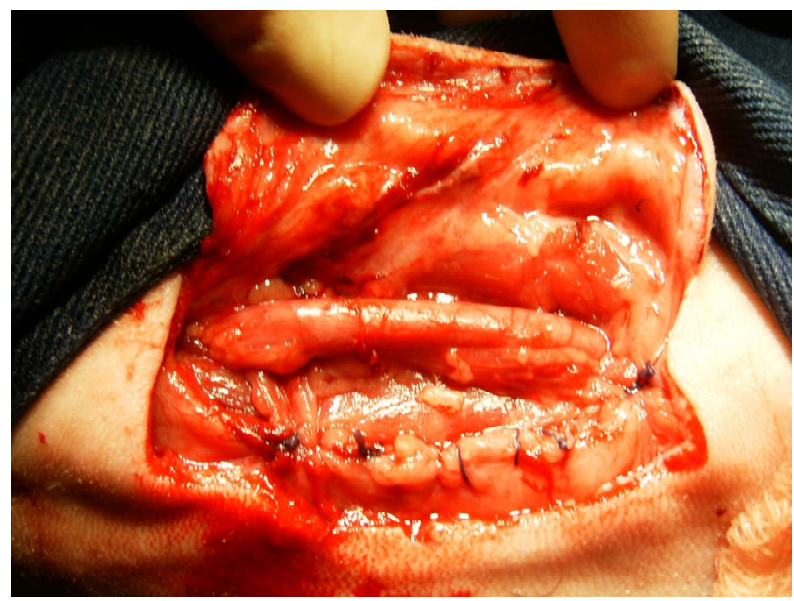

Figure 3. Isolation of the carotid artery and suture of esternocephalic and brachiocephalic muscle fascias covering the jugular vein.

dexamethasone (Dexacort, Marcolab, Sao Sebastiao do Paraiso, Brazil), IV, single dose, to avoid severe edema of the manipulated area, and $5 \mathrm{mg} / \mathrm{kg}$ of enrofloxacin (Zelotril 10\%, National Union Pharmaceutical Chemistry, Embu Guaçu, Brazil), IM, every 24 hours for seven days. Dressings were applied daily with topical $0.1 \%$ polyvinylpyrrolidone (Riodeíne, Rioquímica Pharmaceutical Industry, São José do Rio Preto, Brazil), and the suture was removed approximately ten days after the surgery.

Animals were examined by eco-Doppler ultrasonography (SonoHeart ELITE, SonoSite, USA) with a transducer frequency bandwidth of 4 - $7 \mathrm{MHz}$ after suture removal and after six months of the surgical procedure to assess the patency of carotid blood flow and to measure the distance between the luminal and epidermis.

\section{Results and Discussion}

The technique used on this study was described in horses
[10] and was easily performed without major complications.

Other authors [1,2,7,9] described post-surgical complications of arterial transposition, such as skin necrosis, abscess formation, peri-arterial hematoma, and accidenttal puncture of the carotid artery. None of the animals in this experiment had postoperative complications, possibly by postoperative procedures performed which included the use of dexamethasone, antibiotic therapy, and cryotherapy.

In all animals, blood flow in the carotid artery was present, securing its patency. The distance between the carotid artery and skin was measured and was less than $0.5 \mathrm{~cm}$ in all animals (Figure 4).

According to [1] the effectiveness of a transposition technique is verified by the patency of the carotid artery for a long time, allowing the animals to be used experimentally. In our experiment the carotid artery was evaluated for a period of six months, with maintenance of patency in all animals (100\%), while [9] had 94\% success with other technique.

So far, three years after the transposition, each animal had the carotid catheterized approximately twenty times for sequential collections during experiments involving anesthesia and acid-base imbalances. In all these times the artery was easily identified by its pulsation and no difficulty found in placing the $16 \mathrm{G}$ catheter. With an aneroid sphygmomanometer (Premium Instruments Co. Ltd., Wenzhou., China) arterial pressure could be measured and the blood flow obtained in all catheterizations was similar to the first one, indicating that the artery's lumen was maintained.

The technique described in this work for permanent carotid transposition in sheep proved to be easy to perform, allowing the adequate repositioning of the carotid

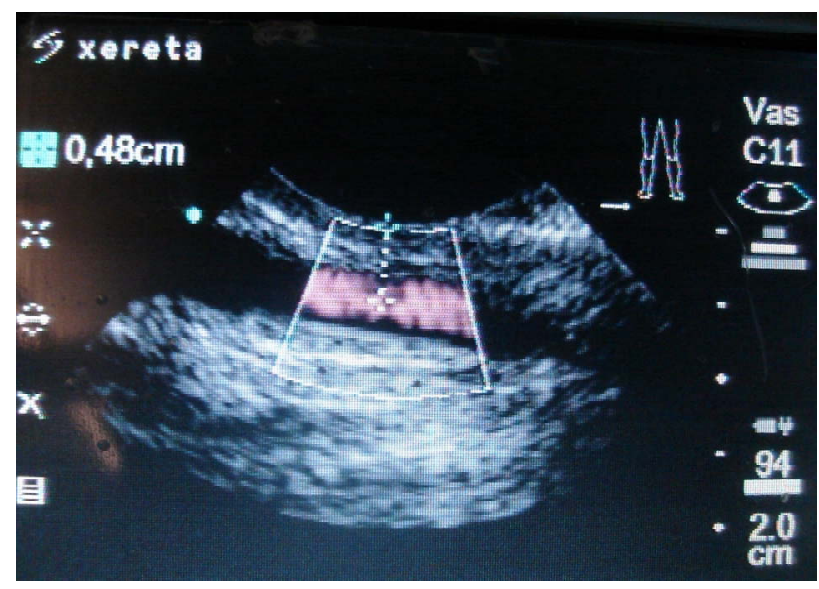

Figure 4. Image obtained with the echo-doppler ultrasound system. Normal blood flow of carotid artery (in red) and the distance between the carotid and skin (dashed line) of 0.48 $\mathrm{cm}$ are shown. Image obtained after suture removal. 
artery for experiments that require multiple and frequent arterial blood collection.

\section{Acknowledgements}

The authors thank the Dean for Research and Graduate College of the University of Brasília, CNPq for fellowships and undergraduate research and FINATEC for financial support.

\section{References}

[1] N. Prassinos, D. Raptopoulos, K. Adamama-Moraitou and A. D. Galatos, "Comparison of Three Different Techniques for Subcutaneous Relocation of the Carotid Artery in Small Ruminant," Journal of Veterinary Medicine Series A, Vol. 48, No. 1, 2001, pp. 15-21.

[2] J. F. Bone, J. Metcalfe and J. T. Parer, "Surgical Preparation of a Carotid Loop in Sheep," American Journal of Veterinary Research, Vol. 23, No. 96, 1962, pp. 11131116.

[3] R. Dueck, J. P. Schroeder, H. R. Parker, M. Rathbun and K. Smolen, "Carotid Artery Exteriorization for Percutaneous Catheterization in Sheep and Dogs," American Journal of Veterinary Research, Vol. 43, No. 5, 1982, pp. 898-901.
[4] W. R. Graham, C. W. Turner Jr. and E. T. Gomez, “A Method for Obtaining Arterial Blood from the Goat," Research Bulletin, University of Missouri, Agricultural Experiment Station, Vol. 260, 1937, pp. 3-15.

[5] K. Hove, “A Permanent Preparation Allowing Measurements of Secretion of Parathyroid Hormone in Conscious Goats,” Journal of Endocrinology, Vol. 90, No. 2, 1981, pp. 295-306. doi:10.1677/joe.0.0900295

[6] S. K. Jha, W. Lumb and R. F. Johnston, "Establishment of Permanent Carotid Loops in Goats,” American Journal of Veterinary Reasearch, Vol. 22, 1961, pp. 948-949.

[7] J. L. Linzell, “Carotid Loops,” American Journal of Veterinary Reasearch, Vol. 24, 1963, pp. 223-224.

[8] G. L. McClymont, "A Note of the Establishment of Carotid Loops in Cattle,” Australian Veterinary Journal, Vol. 26, No. 6, 1950, pp. 138-139. doi:10.1111/j.1751-0813.1950.tb04905.x

[9] M. S. Lagutchik, J. W. Sturgis, D. G. Martin and J. A. Bley, "Review of the Carotid Artery Loop Procedure in Sheep,” Journal of Investigative Surgery, Vol. 5, No. 2, 1992, pp. 79-89. doi:10.3109/08941939209012425

[10] C. L. Sweeney and G. M. Constantinescu, "Vascular Surgery,” In: J. A. Auer, Ed., Equine Surgery, Saunders Company, Philadelphia, 1992, pp. 517-519. 